\title{
Calculating the jet quenching parameter in the plasma of NCYM theory from gauge/gravity duality
}

\author{
Somdeb Chakraborty 1 and Shibaji Roy 2 \\ Saha Institute of Nuclear Physics, 1/AF Bidhannagar, Calcutta-700 064, India
}

\begin{abstract}
A particular decoupling limit of non-extremal (D1, D3) brane bound state system of type IIB string theory is known to give the gravity dual of space-space noncommutative Yang-Mills (NCYM) theory at finite temperature. We use a string probe in this background to compute the jet quenching parameter in a strongly coupled plasma of hot non-commutative Yang-Mills theory in $(3+1)$-dimensions from gauge/gravity duality. We give expressions for the jet quenching parameter for both small and large non-commutativity. For small non-commutativity, we find that the value of the jet quenching parameter gets reduced from its commutative value. The reduction is enhanced with temperature as $T^{7}$ for fixed non-commutativity and fixed 't Hooft coupling. We also give an estimate of the correction due to noncommutativity at the present collider energies like in RHIC or in LHC and find it too small to be detected. We further generalize the results for non-commutative Yang-Mills theories in diverse dimensions.
\end{abstract}

\footnotetext{
${ }^{1}$ E-mail: somdeb.chakraborty@saha.ac.in

${ }^{2}$ E-mail: shibaji.roy@saha.ac.in
} 


\section{Introduction}

Jet quenching parameter [1], denoted by $\hat{q}$, is a measure of the radiative parton energy loss when they interact strongly with the medium (the quark-gluon plasma or QGP), produced in the ultrarelativistic heavy ion collision (for example, $\mathrm{Au}-\mathrm{Au}$ collision in $\mathrm{RHIC}$ or $\mathrm{Pb}$ $\mathrm{Pb}$ collision in LHC), as they traverse through it (see, for example, 2] for reviews on the subject). $\hat{q}$ characterizes the properties of the medium and so by studying it one can gain insight about the properties of QCD matter in the extreme conditions as produced in the collision process. The phenomenological models of medium induced parton energy loss which account for the strong suppression of high- $p_{T}$ hadronic spectra observed in the experiment use a single jet quenching parameter, but this way it is model-dependent and it relies on the perturbative QCD framework [3].

However, there are indications both from experimental data [3, 4] as well as lattice QCD calculations [5] that QGP, at energies not far above the cross-over from the confinement (or the hadronic) phase, is strongly coupled. A model-independent strong coupling calculation of $\hat{q}$ has been performed in [6] using AdS/CFT correspondence [7] and was found to agree [6] reasonably well with the experimental result [3] of RHIC. In this approach one uses weakly coupled string or supergravity theory to calculate quantities in the strongly coupled gauge theory. In the gauge theory $\hat{q}$ can be related to the expectation value of a particular light-like Wilson loop. The latter quantity in string or supergravity theory can be computed by extremizing the area of the string world-sheet whose boundary is the loop in question [8]. Thus one obtains $\hat{q}$ in the strongly coupled gauge theory in a model-independent way. In this spirit, $\hat{q}$ in many different cases have been calculated in [9].

In this paper we compute the jet quenching parameter when the plasma is described by hot non-commutative Yang-Mills theory using gauge/gravity duality. So, in this case the boundary theory is defined on a manifold, where some of the space coordinates are non-commutative. The idea that space-time 3 coordinates can be non-commutative is not new and dates back to Heisenberg and Pauli [11], who first proposed non-commutativity of space-time in order to remove infinities in quantum field theory before renormalization was successful. However, it was Snyder [12] and then Connes [13] who took the idea seriously. Connes and Chamseddine [14] suggested non-commutative geometry as an alternative to Riemannian geometry and obtained a variant of general relativity along with a standard model in non-commutative space - a true geometric unification. Although

\footnotetext{
${ }^{3}$ There is problem with the time coordinate being non-commutative as it is believed to lead to a nonunitary theory [10].
} 
there are various claims [15, 16, 17, 18] for the lower bound of the non-commutativity scale, experimentally there is no evidence for or against it. In string [19, 20, 21] or M-theory [22] non-commutative gauge theory arises quite naturally in a particular low energy limit. Just like ordinary Yang-Mills theory appears on the boundary of AdS-space, a low energy decoupling limit of D3-brane background, a space-space non-commutative Yang-Mills theory appears in a particular low energy limit of (D1, D3) bound state [23] background of type IIB string theory. In this case the world-volume of D3-brane contains a magnetic field which in the decoupling limit becomes asymptotically large [19] and makes some of the spatial directions non-commutative [24]. So, it would be natural to ask how non-commutativity affects the jet quenching parameter of the plasma described by ordinary Yang-Mills theory and this is the question we would like to explore in this paper.

Due to the star product and the associated complications, the perturbative calculation of the jet quenching parameter in NCYM theory would be quite involved. However, like in the commutative case, assuming the plasma to be strongly coupled, the natural approach to do this calculation would be the gauge/gravity duality once we know the gravity dual of NCYM theory. We take this approach where the dual gravity background for NCYM theory is known to be a particular decoupling limit of (D1, D3) bound state of type IIB string theory. We use a string probe in this background and extremize the NambuGoto string world-sheet action in a static gauge and by gauge/gravity duality obtain the expectation value of a particular light-like Wilson loop [8]. This, in turn, determines the jet quenching parameter [2] of the plasma of hot NCYM theory. In obtaining the final form of $\hat{q}_{\mathrm{NCYM}}$ we need to regularize an integral which we will discuss in appropriate place. We obtain $\hat{q}_{\mathrm{NCYM}}$ for both small and large non-commutativity. For small non-commutativity, the jet quenching parameter $\hat{q}_{\mathrm{NCYM}}$, on top of the value $\sim\left(\sqrt{\hat{\lambda}} T^{3}\right)$, gets corrected by $\sim-\left(\hat{\lambda}^{3 / 2} T^{7} \theta^{2}\right)$, where $T$ is the temperature of the plasma, $\hat{\lambda}$ is the 't Hooft coupling in the NCYM theory and $\theta$ is the non-commutativity parameter. So, the jet quenching (or the parton energy loss) gets reduced by the non-commutative effect and also the reduction gets enhanced with increasing temperature as $T^{7}$. On the other hand, for large non-commutativity the jet quenching varies as $\sim 1 /\left(\sqrt{\hat{\lambda}} T \theta^{2}\right)$. So, the jet quenching in this case varies inversely with temperature. We then generalize $\hat{q}_{\mathrm{NCYM}}$ for NCYM theories in other dimensions.

Jet quenching in (3+1)-dimensional NCYM theory has also been considered recently in [25] and [26]. In 25] a (3+1)-dimensional non-commutative, non-relativistic Yang-Mills theory has been considered following [27]. So, when the non-relativistic parameter is set to zero, the jet quenching result must coincide with the result obtained in this paper. 
However, the authors gave only a formal expression and did not discuss the subtleties involved in the exact calculation performed in this paper. On the other hand [26] discussed NCYM theory from Sakai-Sugimoto model and the background considered there is D4brane instead of D3-brane. However, the conclusion found there is quite similar to what we find here.

This paper is organized as follows. In section 2, we compute the jet quenching parameter in $(3+1)$-dimensional NCYM theory and the dual gravity background is given by a decoupling limit of (D1, D3) brane bound state system. We generalize this result to include the jet quenching calculation in other dimensions in section 3. The dual gravity background in this case is given by the NCYM decoupling limit of ( $\mathrm{D}(p-2), \mathrm{D} p)$ brane bound state system. Our conclusion is presented in section 4 .

\section{Jet quenching for $(3+1)$-dim. NCYM theory}

In this section we will calculate the jet quenching parameter for $(3+1)$-dimensional NCYM theory from a light-like Wilson loop using gauge/gravity duality. The dual gravity background for NCYM theory is the NCYM decoupling limit of non-extremal (D1, D3) bound state system of type IIB string theory. The various field configurations of non-extremal (D1, D3) brane bound state solution are given as [28],

$$
\begin{aligned}
d s^{2} & =H^{-\frac{1}{2}}\left(-f(d t)^{2}+\left(d x^{1}\right)^{2}+\frac{H}{F}\left(\left(d x^{2}\right)^{2}+\left(d x^{3}\right)^{2}\right)\right)+H^{\frac{1}{2}}\left(\frac{d r^{2}}{f}+r^{2} d \Omega_{5}^{2}\right) \\
e^{2 \phi} & =g_{s}^{2} \frac{H}{F}, \quad B_{23}=\frac{\tan \alpha}{F} \\
A_{01} & =\frac{1}{g_{s}}\left(H^{-1}-1\right) \sin \alpha \operatorname{coth} \varphi, \quad A_{0123}=\frac{1}{g_{s}} \frac{(1-H)}{F} \cos \alpha \operatorname{coth} \varphi+\mathrm{T} . \mathrm{T} .
\end{aligned}
$$

where the various functions appearing above are,

$$
f=1-\frac{r_{0}^{4}}{r^{4}}, \quad H=1+\frac{r_{0}^{4} \sinh ^{2} \varphi}{r^{4}}, \quad F=1+\frac{r_{0}^{4} \cos ^{2} \alpha \sinh ^{2} \varphi}{r^{4}}
$$

The metric in (11) is given in the string frame. Note that D3-branes lie along $x^{1}, x^{2}, x^{3}$ and D1-branes lie along $x^{1}$. The angle $\alpha$ measures the relative numbers of D1 and D3-branes and is defined as, $\cos \alpha=N / \sqrt{N^{2}+M^{2}}$, where $N$ is the number of D3-branes and $M$ is the number of D1-branes per unit co-dimension two surface transverse to D1-branes. Also $\varphi$ is the boost parameter and $r_{0}$ is the radius of the horizon of the non-extremal (D1, D3) bound state solution. $\phi$ is the dilaton and $g_{s}$ is the string coupling constant. $B_{23}$ is the NSNS 2-form which is responsible for the appearance of non-commutativity in the 
decoupling limit. $A_{01}$ and $A_{0123}$ are the RR 2-form and 4-form respectively. T.T. denotes a term involving the transverse part of the brane to make the corresponding field-strength self-dual. However, we do not need its explicit form for our discussion.

The NCYM decoupling limit [19] is a low energy limit for which we zoom into the region

$$
r_{0}<r \sim r_{0} \sqrt{\sinh \varphi \cos \alpha} \ll r_{0} \sqrt{\sinh \varphi}
$$

It is clear from (3) that $\varphi$ is very large and the angle $\alpha$ is close to $\pi / 2$. Now in this approximation (3), we get

$$
H \approx \frac{r_{0}^{4} \sinh ^{2} \varphi}{r^{4}}, \quad \frac{H}{F} \approx \frac{1}{\cos ^{2} \alpha\left(1+a^{4} r^{4}\right)} \equiv \frac{h}{\cos ^{2} \alpha}
$$

where,

$$
h=\frac{1}{1+a^{4} r^{4}}, \quad \text { with, } \quad a^{4}=\frac{1}{r_{0}^{4} \sinh ^{2} \varphi \cos ^{2} \alpha}
$$

It is clear from (11) that the asymptotic value of the $B$-field is $\tan \alpha$ and since $\alpha \rightarrow \pi / 2$ in the NCYM limit, the $B$-field becomes very large. Note that the non-vanishing component of the $B$-field is $B_{23}$ which gives rise to a magnetic field in the D3-brane world-volume and is responsible for making $x^{2}$ and $x^{3}$ directions non-commutative. Using (4) we rewrite the metric in (1) in light-cone coordinate as,

$$
\begin{aligned}
d s^{2}= & \frac{r^{2}}{r_{0}^{2} \sinh \varphi}\left[-(1+f) d x^{+} d x^{-}+\frac{1}{2}(1-f)\left[\left(d x^{+}\right)^{2}+\left(d x^{-}\right)^{2}\right]\right. \\
& \left.+h\left[\left(d x^{2}\right)^{2}+\left(d x^{3}\right)^{2}\right]\right]+\frac{r_{0}^{2} \sinh \varphi}{r^{2}} \frac{d r^{2}}{f}+r_{0}^{2} \sinh \varphi d \Omega_{5}^{2} \\
= & G_{\mu \nu} d x^{\mu} d x^{\nu}
\end{aligned}
$$

where we have defined $x^{ \pm}=\left(t \pm x^{1}\right) / \sqrt{2}$. The function $h$ is as given in (5) and also in writing (6) we have rescaled the coordinates as, $x^{2,3} \rightarrow \cos \alpha x^{2,3}$. The above metric, along with the other field configurations (in the NCYM limit) in (1), is the gravity dual of (3+1)-dimensional NCYM theory at finite temperature.

Now to compute the expectation value of the (light-like) Wilson loop $(\mathcal{C})$ we extremize the action $(S(\mathcal{C}))$ of the string world-sheet whose boundary is the mentioned loop [6, 29]. The Nambu-Goto action for the string world-sheet is

$$
S=\frac{1}{2 \pi \alpha^{\prime}} \int d \tau d \sigma \sqrt{\operatorname{det} g_{a b}}
$$

where $g_{a b}$ is the induced metric on the world-sheet and is given as,

$$
g_{a b}=\frac{\partial x^{\mu}}{\partial \xi^{a}} \frac{\partial x^{\nu}}{\partial \xi^{b}} G_{\mu \nu}
$$


Here $G_{\mu \nu}$ is the background metric given in (6) and $\xi^{a, b}, a, b=0,1$ are the world-sheet coordinates $\tau=\xi^{0}$ and $\sigma=\xi^{1}$. Due to reparametrization invariance of the action (7) we can set $\tau=x^{-}$and $\sigma=x^{2}$. The length of the rectangular loop $\mathcal{C}$ along $x^{2}$ and $x^{-}$ are $L$ and $L^{-}$respectively and we assume $L^{-} \gg L$. As a result the surface is invariant under $\tau$-translation and we have $x^{\mu}(\tau, \sigma)=x^{\mu}(\sigma)$. Furthermore, the Wilson loop lies at $x^{+}=$constant and $x^{3}=$ constant. Note that one of the sides of the rectangular Wilson loop is chosen along a non-commutative direction $\left(x^{2}\right)$ so that the jet quenching parameter evaluated from this Wilson loop will be affected by non-commutativity. The radial coordinate $r(\sigma)$ gives the string embedding and we impose the condition that the world sheet has $\mathcal{C}$ as its boundary, i.e., $r( \pm L / 2)=r_{0} \Lambda$, for some finite $\Lambda$. We will take $\Lambda \rightarrow \infty$ at the end. The action (17) now reduces to,

$$
S=\frac{\sqrt{2} L^{-}}{2 \pi \alpha^{\prime} \sinh \varphi} \int_{0}^{L / 2} d \sigma\left[\frac{1}{1+a^{4} r^{4}}+\frac{r_{0}^{4} \sinh ^{2} \varphi}{r^{4}-r_{0}^{4}}\left(r^{\prime}\right)^{2}\right]^{\frac{1}{2}}
$$

where $r^{\prime}=\partial_{\sigma} r$. Defining new dimensionless variables $y=r / r_{0}, \tilde{\sigma}=\sigma /\left(r_{0} \sinh \varphi\right)$ and $\ell=L /\left(r_{0} \sinh \varphi\right)$, we can rewrite the action as,

$$
S=\frac{\sqrt{2} L^{-} r_{0}}{2 \pi \alpha^{\prime}} \int_{0}^{\ell / 2} d \sigma\left[\frac{1}{1+a^{4} r_{0}^{4} y^{4}}+\frac{\left(y^{\prime}\right)^{2}}{y^{4}-1}\right]^{\frac{1}{2}}
$$

Note that in writing (10) we have omitted the 'tilde' from $\sigma$ for convenience. The equation of motion following from (10) for $y(\sigma)$ is given as,

$$
y^{\prime}=\left[1-q_{0}^{2}\left(1+a^{4} r_{0}^{4} y^{4}\right)\right]^{\frac{1}{2}} \frac{\sqrt{y^{4}-1}}{q_{0}\left(1+a^{4} r_{0}^{4} y^{4}\right)}
$$

where $q_{0}$ is an integration constant. From the first factor in (11) we have $q_{0}<1 /(1+$ $\left.a^{4} r_{0}^{4} y^{4}\right)^{\frac{1}{2}}$ for all values of $y$. In fact, $q_{0}$ has more stringent restriction to be mentioned later. So, the above equation has a solution 4 such that $y$ starts from $\Lambda$ and then comes all the way down to $y=1$ where there is a turning point with $y^{\prime}=0$ and goes back again to $\Lambda$. Integrating (11) we obtain,

$$
\ell=2 \int_{0}^{\ell / 2} d \sigma=2 q_{0} \int_{1}^{\Lambda} d y \frac{1+a^{4} r_{0}^{4} y^{4}}{\sqrt{\left(y^{4}-1\right)\left[1-q_{0}^{2}\left(1+a^{4} r_{0}^{4} y^{4}\right)\right]}}
$$

Now since $\ell=L /\left(r_{0} \sinh \varphi\right)$ is very small compared to any other length scale of the problem, it implies from (12) that $q_{0}$ must be very small, i.e., $q_{0} \ll 1 / \sqrt{1+a^{4} r_{0}^{4} \Lambda^{4}}$ and

\footnotetext{
${ }^{4}$ Note that here we discard another solution at UV corresponding to the surface at infinity. Since $\hat{q}$ is a property of the thermal medium and does not describe UV physics, the surface at infinity is not physically relevant [6].
} 
so, we can expand (12) in powers of $q_{0}$ and from there we formally obtain its value as,

$$
q_{0}=\frac{\ell}{2}\left[\int_{1}^{\Lambda} d y \frac{1+a^{4} r_{0}^{4} y^{4}}{\sqrt{y^{4}-1}}\right]^{-1}
$$

Substituting (11) in the action (10) and then again expanding in powers of $q_{0}$, we obtain

$$
S-S_{0}=\frac{\sqrt{2} L^{-} r_{0} q_{0}^{2}}{4 \pi \alpha^{\prime}} \int_{1}^{\Lambda} d y \frac{1+a^{4} r_{0}^{4} y^{4}}{\sqrt{y^{4}-1}}=\frac{\sqrt{2} L^{-} r_{0} \ell^{2}}{16 \pi \alpha^{\prime}}\left[\int_{1}^{\Lambda} d y \frac{1+a^{4} r_{0}^{4} y^{4}}{\sqrt{y^{4}-1}}\right]^{-1}
$$

where in the last expression we have used (13). In the above $S_{0}$ denotes the action for the world-sheet of two free strings (or the self energy of the quark-antiquark pair). Here we would like to remark that the integral in the square bracket in (14) is actually divergent if we take the boundary $(\Lambda)$ where the NCYM theory lives to $\infty$. The evaluation of the action here differs from the commutative case. In the commutative case the action, after subtracting the self-energy of the quarks, i.e., (14) becomes finite and this can be seen if we put $a^{4} r_{0}^{4}$, which is a measure of non-commutativity (to be discussed later), to zero. However, for the non-commutative case, the action (14) is still divergent if we put $\Lambda \rightarrow \infty$. The reason for this divergence is that in the non-commutative case the NCYM theory does not live at $r=\propto 5$, the usual boundary of the $\mathrm{AdS}_{5}$-space, but rather lives on a surface which is at a finite distance before $r=\infty$. Instead of directly evaluating this distance what we will do is that we will first evaluate the integral in the square bracket of (14) for finite $\Lambda$ and then subtract the part which is divergent when we put $\Lambda \rightarrow \infty$. This way we regularize the integral in order to give any meaning to the extremized action 6 . Once the subtraction is made the NCYM theory can be considered to be living effectively

\footnotetext{
${ }^{5}$ This is implicit in the quark-antiquark potential calculation done in [20] (see also [30]). There it was not possible to fix the position of the string at infinity since a small perturbation would change it violently. So, the calculation was performed by going to a conjugate 'momentum' variable and the energy was found to be divergent. A finite answer was obtained only after subtracting the divergent part. This, in turn, implies that the boundary screen is not at infinity but at a finite radial distance [30.

${ }^{6}$ There are two ways to describe the finiteness of the integral in the action (14). Either we take $\Lambda$ to be finite in which case it is obviously finite (in this case the integral can be evaluated only if we know the exact position of the boundary) or we take $\Lambda$ to be infinite and subtract the unique divergent part (as explicitly calculated below) of the integral and obtain a finite result. In the former case the boundary is at a finite radial distance, but for the latter case it is at infinity. But, effectively, they describe the same thing. Here we have adopted the second approach.
} 
on the usual $r=\infty$ boundary. So, we first evaluate the integral for finite $\Lambda$ as follows,

$$
\begin{aligned}
\int_{1}^{\Lambda} d y \frac{1+a^{4} r_{0}^{4} y^{4}}{\sqrt{y^{4}-1}}= & -\Lambda \sqrt{\Lambda^{4}-1}+\frac{1}{3}\left(3+a^{4} r_{0}^{4}\right) \frac{\sqrt{\pi} \Gamma\left(\frac{5}{4}\right)}{\Gamma\left(\frac{3}{4}\right)} \\
& +\frac{1}{3}\left(3+a^{4} r_{0}^{4}\right) \Lambda_{2}^{3} F_{1}\left(-\frac{3}{4}, \frac{1}{2} ; \frac{1}{4} ; \frac{1}{\Lambda^{4}}\right)
\end{aligned}
$$

where ${ }_{2} F_{1}\left(a, b ; c ; 1 / \Lambda^{4}\right)$ is a hypergeometric function. For large $\Lambda$ it has an expansion

$$
{ }_{2} F_{1}\left(a, b ; c ; \frac{1}{\Lambda^{4}}\right)=1+\frac{a b}{c} \frac{1}{\Lambda^{4}}+\frac{a(a+1) b(b+1)}{2 c(c+1)} \frac{1}{\Lambda^{8}}+\cdots
$$

Using the above expansion (16) in the rhs of (15) and finally setting $\Lambda \rightarrow \infty$, we find that apart from a finite part the above integral has a single divergent piece of the form $\left(a^{4} r_{0}^{4} / 3\right) \Lambda^{3}$ and all other terms vanish. So, removing the divergent part we get the regularized integral as,

$$
\int_{1}^{\infty} d y \frac{1+a^{4} r_{0}^{4} y^{4}}{\sqrt{y^{4}-1}}=\left(1+\frac{a^{4} r_{0}^{4}}{3}\right) \frac{\sqrt{\pi} \Gamma\left(\frac{5}{4}\right)}{\Gamma\left(\frac{3}{4}\right)}
$$

Substituting (17) in (14) yields,

$$
S-S_{0}=\frac{\sqrt{2} L^{-} r_{0} \ell^{2}}{16 \pi \alpha^{\prime}} \frac{\Gamma\left(\frac{3}{4}\right)}{\sqrt{\pi} \Gamma\left(\frac{5}{4}\right)}\left(1+\frac{a^{4} r_{0}^{4}}{3}\right)^{-1}
$$

Now to extract the jet quenching parameter $\hat{q}_{\mathrm{NCYM}}$ from (18), we use the definition,

$$
e^{-2\left(S-S_{0}\right)}=\langle W(\mathcal{C})\rangle=e^{-\frac{1}{4 \sqrt{2}} \hat{q}_{\mathrm{NCYM}} L^{-} L^{2}}
$$

where $W(\mathcal{C})$ denotes the Wilson loop and the factor 2 in front of $\left(S-S_{0}\right)$ denotes that we are dealing with adjoint Wilson loop. Thus from (18) we have,

$$
\hat{q}_{\mathrm{NCYM}}=\frac{r_{0}}{\pi \alpha^{\prime} r_{0}^{2} \sinh ^{2} \varphi} \frac{\Gamma\left(\frac{3}{4}\right)}{\sqrt{\pi} \Gamma\left(\frac{5}{4}\right)}\left(1+\frac{a^{4} r_{0}^{4}}{3}\right)^{-1}
$$

where we have used $\ell=L /\left(r_{0} \sinh \varphi\right)$. Note that here we have expressed the jet quenching parameter in terms of the paramaters of string theory or supergravity. However, since the jet quenching parameter is a property of a gauge theory, we must express it in terms of the parameters of the NCYM theory and we can do that by using the gauge/gravity dictionary [20]. The temperature of the non-extremal (D1, D3) bound state which by gauge/gravity duality is the temperature of the NCYM theory can be obtained from the metric in (11) and has the form,

$$
T=\frac{1}{\pi r_{0} \cosh \varphi} \approx \frac{1}{\pi r_{0} \sinh \varphi}
$$


where in the last expression we have used the fact that in the decoupling limit (3), $\varphi$ is large. Also from the charge of the D3-brane we have

$$
r_{0}^{4} \sinh ^{2} \varphi=2 \hat{\lambda} \alpha^{\prime 2}
$$

Here $\hat{\lambda}=\hat{g}_{\mathrm{YM}}^{2} N$ is the 't Hooft coupling of the NCYM theory and $\hat{g}_{\mathrm{YM}}$ is the NCYM coupling, with $N$ being the number of D3-branes or the rank of the gauge group. The NCYM 't Hooft coupling is related to the ordinary 't Hooft coupling by $\lambda=\left(\alpha^{\prime} / \theta\right) \hat{\lambda}$, where $\theta$ is the non-commutativity parameter defined by $\left[x^{2}, x^{3}\right]=i \theta$. Here $\theta$ is a finite parameter and in the decoupling limit as $\alpha^{\prime} \rightarrow 0, \hat{\lambda}$ remains finite. Using (21) and (22) we obtain,

$$
\sinh \varphi=\frac{1}{\pi^{2} \sqrt{2 \hat{\lambda}} T^{2} \alpha^{\prime}}, \quad \text { and } \quad r_{0}=\pi \sqrt{2 \hat{\lambda}} T \alpha^{\prime}
$$

Also we have

$$
a^{4} r_{0}^{4}=\frac{1}{\sinh ^{2} \varphi \cos ^{2} \alpha}=\pi^{4}(2 \hat{\lambda}) T^{4} \theta^{2}
$$

Note that in the above we have used the decoupling limit $\cos \alpha=\alpha^{\prime} / \theta$ and as $\alpha^{\prime} \rightarrow 0$, $\alpha \rightarrow \pi / 2$ as we mentioned earlier. Also, from (24) we notice that since $a^{2} r_{0}^{2}$ is proportional to $\theta$, therefore, $a r_{0}$ is a measure of non-commutativity. Now using (23) and (24) in (20) we find that for small non-commutativity $\left(\operatorname{ar}_{0} \sim \theta \ll 1\right)$ the jet quenching parameter in the NCYM theory has the form,

$$
\hat{q}_{\mathrm{NCYM}}=\frac{\pi^{\frac{3}{2}} \Gamma\left(\frac{3}{4}\right)}{\Gamma\left(\frac{5}{4}\right)} \sqrt{\hat{\lambda}} T^{3}\left[1-\frac{\pi^{4} \hat{\lambda} T^{4} \theta^{2}}{3}+O\left(\theta^{4}\right)\right]
$$

We notice from (25) that when the non-commutativity parameter $\theta$ is put to zero, we recover the jet quenching of the ordinary Yang-Mills plasma (note that in this case the NCYM 't Hooft coupling $\hat{\lambda}$ becomes equal to ordinary 't Hooft coupling $\lambda$ and also in writing (25) we have replaced $2 \hat{\lambda}$ by $\hat{\lambda}$ to match the commutative results in [6]. This difference in a factor of 2 is just a convention as mentioned in [29].). But in the presence of non-commutativity we find that the jet quenching gets reduced from its commutative value and the reduction gets enhanced with temperature as $T^{7}$, keeping the other parameters fixed. The reduction in the jet quenching for the non-commutative case can be intuitively understood as non-commutativity introduces a non-locality in space due to space uncertainty and there is no point-like interaction among the partons. So, the parton energy loss would be less in this case.

We can try to estimate the correction (the second term in (25)) in the jet quenching due to non-commutativity from the experimental bound on the non-commutativity scale. In 
the literature various disparate experimental bounds on $\theta$ has been obtained from various physical considerations. The bound on $\theta$ has been claimed to be $\sim(1-10 \mathrm{TeV})^{-2}$ in [15], whereas, it is $\sim\left(10^{12}-10^{13} \mathrm{GeV}\right)^{-2}$ in [16] or even stronger $\sim\left(10^{15} \mathrm{GeV}\right)^{-2}$ in [17]. In theories of gravity it can be of the order of Planck scale $\sim\left(10^{19} \mathrm{GeV}\right)^{-2}$ [18]. It is clear that in all these cases except the first one there is no hope of getting a significant correction due to non-commutativity in collider experiments. At RHIC collision energy $\sim 200 \mathrm{GeV}$ where the temperature attained by QGP is $\sim 300 \mathrm{MeV}$, even the first case does not give a significant correction $\left(\pi^{4} \hat{\lambda} T^{4} \theta^{2} / 3 \sim 4.96 \times 10^{-12}\right.$ taking $\hat{\lambda}=6 \pi$ and $T=300$ $\mathrm{MeV}$ relevant for the $\mathrm{Au}-\mathrm{Au}$ collision at RHIC and taking $\theta=1 \mathrm{TeV}^{-2}$ ) compared to the leading order term. At LHC where the collision energy would be much higher, the temperature of the QGP may rise and is expected to go up to $(1-10) \mathrm{GeV}$. In that case the correction to the jet quenching due to non-commutativity can be estimated to be $\pi^{4} \hat{\lambda} T^{4} \theta^{2} / 3 \sim\left(6.12 \times 10^{-6}-6.12 \times 10^{-10}\right)$, still too low to be detected. Conversely, in order to get a $10 \%$ correction on the jet quenching parameter due to non-commutativity, the temperature of the plasma would have to be $T \sim 200 \mathrm{GeV}$.

For large non-commutativity $\left(a r_{0} \sim \theta \gg 1\right)$, on the other hand, the jet quenching parameter (20) takes the form,

$$
\hat{q}_{\mathrm{NCYM}}=\frac{3 \Gamma\left(\frac{3}{4}\right)}{\pi^{\frac{5}{2}} \Gamma\left(\frac{5}{4}\right)} \frac{1}{\sqrt{\hat{\lambda}} T \theta^{2}}\left[1-\frac{3}{\pi^{4} \hat{\lambda} T^{4} \theta^{2}}+O\left(\frac{1}{\theta^{4}}\right)\right]
$$

We thus find that for large non-commutativity, the jet quenching varies inversely with temperature and also inversely with the square-root of the NCYM 't Hooft coupling. In this case we can not recover the commutative result as the non-commutativity parameter is large. This completes our derivation and the discussion of the jet quenching parameter for the plasma of $(3+1)$-dimensional NCYM theory.

\section{Jet quenching for NCYM theories in other dimen- sions}

We can easily generalize the above calculation of the jet quenching parameter in $(3+1)$ dimensions to NCYM theories in other dimensions. The procedure is exactly similar to the $(3+1)$-dimensional case and so we will be brief here. For generalization, the gravity

\footnotetext{
${ }^{7}$ We have taken the 't Hooft coupling of the NCYM theory to be the same as that of the commutative theory, although there is no concrete reason for this. This is taken just for the estimate. Actually these two couplings are related as given earlier and as $\alpha^{\prime} \rightarrow 0, \lambda \rightarrow 0$, but $\hat{\lambda}$ remains finite. We have taken this finite value to be $6 \pi$ for better comparison.
} 
background we use is $(\mathrm{D}(p-2), \mathrm{D} p)$ brane bound state system of type II string theory and is given as [28],

$$
\begin{aligned}
d s^{2}= & H^{-\frac{1}{2}}\left(-f(d t)^{2}+\sum_{i=1}^{p-2}\left(d x^{i}\right)^{2}+\frac{H}{F}\left(\left(d x^{p-1}\right)^{2}+\left(d x^{p}\right)^{2}\right)\right) \\
& +H^{\frac{1}{2}}\left(\frac{d r^{2}}{f}+r^{2} d \Omega_{8-p}^{2}\right) \\
e^{2 \phi}= & g_{s}^{2} \frac{H^{\frac{5-p}{2}}}{F}, \quad B_{p-1, p}=\frac{\tan \alpha}{F} \\
A_{01 \ldots p-2}= & \frac{1}{g_{s}}\left(H^{-1}-1\right) \sin \alpha \operatorname{coth} \varphi, \quad A_{01 \ldots p}=\frac{1}{g_{s}} \frac{(1-H)}{F} \cos \alpha \operatorname{coth} \varphi
\end{aligned}
$$

where the various functions appearing above are,

$$
f=1-\frac{r_{0}^{7-p}}{r^{7-p}}, \quad H=1+\frac{r_{0}^{7-p} \sinh ^{2} \varphi}{r^{7-p}}, \quad F=1+\frac{r_{0}^{7-p} \cos ^{2} \alpha \sinh ^{2} \varphi}{r^{7-p}}
$$

Here $\mathrm{D} p$-branes lie along $x^{1}, x^{2}, \ldots, x^{p}$ and $\mathrm{D}(p-2)$-branes lie along $x^{1}, x^{2}, \ldots, x^{p-2}$. $\alpha$ and $\varphi$ are as defined before, but now for $\mathrm{D}(p-2), \mathrm{D} p$ branes. The NCYM decoupling limit is 31,32 ]

$$
r_{0}<r \sim r_{0} \sinh ^{\frac{2}{7-p}} \varphi \cos ^{\frac{2}{7-p}} \alpha \ll r_{0} \sinh ^{\frac{2}{7-p}} \varphi
$$

In this approximation,

$$
H \approx \frac{r_{0}^{7-p} \sinh ^{2} \varphi}{r^{7-p}}, \quad \frac{H}{F} \approx \frac{1}{\cos ^{2} \alpha\left(1+a^{7-p} r^{7-p}\right)}
$$

where,

$$
h=\frac{1}{1+a^{7-p} r^{7-p}}, \quad \text { with } \quad a^{7-p}=\frac{1}{r_{0}^{7-p} \sinh ^{2} \varphi \cos ^{2} \alpha}
$$

Now using (30) and (31) we can rewrite the metric (27) in light-cone coordinates as follows,

$$
\begin{aligned}
d s^{2}= & \frac{r^{\frac{7-p}{2}}}{r_{0}^{\frac{7-p}{2}} \sinh \varphi}\left[-(1+f) d x^{+} d x^{-}+\frac{1}{2}(1-f)\left[\left(d x^{+}\right)^{2}+\left(d x^{-}\right)^{2}\right]+\sum_{i=2}^{p-2}\left(d x^{i}\right)^{2}\right. \\
& \left.\quad+h\left[\left(d x^{p-1}\right)^{2}+\left(d x^{p}\right)^{2}\right]\right]+\frac{r_{0}^{\frac{7-p}{2}} \sinh \varphi}{r^{\frac{7-p}{2}}} \frac{d r^{2}}{f}+\frac{r_{0}^{\frac{7-p}{2}}}{r^{\frac{3-p}{2}}} \sinh \varphi d \Omega_{8-p}^{2} \\
= & G_{\mu \nu} d x^{\mu} d x^{\nu}
\end{aligned}
$$

In writing (32) we have rescaled $x^{p-1, p} \rightarrow \cos \alpha x^{p-1, p}$ and these are the two noncommutative directions with $\left[x^{p-1}, x^{p}\right]=i \theta$. This metric along with the other field configurations in the NCYM limit is the gravity dual of $(p+1)$-dimensional NCYM theory. It should be 
noted here that $p$ in the above configuration (32) is greater than 2 and since the brane configuration in general develops instability for $p>4$, so, strictly speaking, the results below are sound only for $p=3,4$. Now setting $\tau=x^{-}$and $\sigma=x^{p}$, we can evaluate the Nambu-Goto action exactly as before and we obtain in this case,

$$
S-S_{0}=\frac{\sqrt{2} L^{-} r_{0} q_{0}^{2}}{4 \pi \alpha^{\prime}} \int_{1}^{\Lambda} d y \frac{1+a^{7-p} r_{0}^{7-p} y^{7-p}}{\sqrt{y^{7-p}-1}}=\frac{\sqrt{2} L^{-} r_{0} \ell^{2}}{16 \pi \alpha^{\prime}}\left[\int_{1}^{\Lambda} d y \frac{1+a^{7-p} r_{0}^{7-p} y^{7-p}}{\sqrt{y^{7-p}-1}}\right]^{-1}
$$

Again the integral in the square bracket is divergent for $\Lambda \rightarrow \infty$. So, as before, we regularize it and the regularized integral has the value,

$$
\int_{1}^{\infty} d y \frac{1+a^{7-p} r_{0}^{7-p} y^{7-p}}{\sqrt{y^{7-p}-1}}=\left(1+\frac{2 a^{7-p} r_{0}^{7-p}}{9-p}\right) a_{p}, \quad \text { with } \quad a_{p}=\frac{\sqrt{\pi}}{7-p} \frac{\Gamma\left(\frac{5-p}{2(7-p)}\right)}{\Gamma\left(\frac{6-p}{7-p}\right)}
$$

Using (34) in (33) and also using the definition (19) we extract the form of the jet quenching parameter for the plasma of $(p+1)$-dimensional NCYM theories as,

$$
\hat{q}_{\mathrm{NCYM}}=\frac{r_{0}}{\pi \alpha^{\prime} r_{0}^{2} \sinh ^{2} \varphi a_{p}}\left(1+\frac{2 a^{7-p} r_{0}^{7-p}}{9-p}\right)^{-1}
$$

Now to express $\hat{q}_{\mathrm{NCYM}}$ in terms of the parameters of the NCYM theory we use the following relations [32, 33, 31],

$$
T=\frac{7-p}{4 \pi r_{0} \sinh \varphi}, \quad r_{0}^{7-p} \sinh ^{2} \varphi=d_{p} \hat{\lambda} \alpha^{15-p}, \quad \text { with } \quad d_{p}=2^{7-2 p} \pi^{\frac{9-3 p}{2}} \Gamma\left(\frac{7-p}{2}\right)
$$

Using (36) in (35) we find the form of the jet quenching parameter as,

$$
\hat{q}_{\mathrm{NCYM}}=\frac{8 \sqrt{\pi} \Gamma\left(\frac{6-p}{7-p}\right)}{\Gamma\left(\frac{5-p}{14-2 p}\right)} b_{p}^{\frac{1}{2}} T^{2}(\sqrt{\hat{\lambda}} T)^{\frac{2}{5-p}}\left[1+\frac{8 \pi^{2}}{9-p} b_{p} T^{2}(\sqrt{\hat{\lambda}} T)^{\frac{4}{5-p}} \theta^{2}\right]^{-1}
$$

where $b_{p}=\left[\left\{2^{16-3 p} \pi^{(13-3 p) / 2} \Gamma((7-p) / 2)\right\} /(7-p)^{7-p}\right]^{2 /(5-p)}$. From the above expression it may seem that since the jet quenching parameter in $(p+1)$-dimensions depends, in general, on $p$, so it is non-universal. However, by defining a $T$-dependent dimensionless effective 't Hooft coupling $\hat{\lambda}_{\text {eff }}(T)=\hat{\lambda} T^{p-3}$ [29] we can rewrite the jet quenching parameter 
for small non-commutativity as,

$$
\begin{aligned}
\hat{q}_{\mathrm{NCYM}}= & \frac{8 \sqrt{\pi} \Gamma\left(\frac{6-p}{7-p}\right)}{\Gamma\left(\frac{5-p}{14-2 p}\right)} b_{p}^{\frac{1}{2}} \hat{\lambda}_{\text {eff }}^{\frac{p-3}{2(5-p)}}(T) \sqrt{\hat{\lambda}_{\mathrm{eff}}(T)} T^{3} \\
& \times\left[1-\frac{8 \pi^{2}}{9-p} b_{p} \hat{\lambda}_{\mathrm{eff}}^{\frac{p-3}{5-p}}(T) \hat{\lambda}_{\mathrm{eff}}(T) T^{4} \theta^{2}+O\left(\theta^{4}\right)\right] \\
= & \frac{8 \sqrt{\pi} \Gamma\left(\frac{6-p}{7-p}\right)}{\Gamma\left(\frac{5-p}{14-2 p}\right)} \sqrt{\hat{a}\left(\hat{\lambda}_{\mathrm{eff}}\right)} \sqrt{\hat{\lambda}_{\mathrm{eff}}(T)} T^{3}\left[1-\frac{8 \pi^{2}}{9-p} \hat{a}\left(\hat{\lambda}_{\mathrm{eff}}\right) \hat{\lambda}_{\mathrm{eff}}(T) T^{4} \theta^{2}+O\left(\theta^{4}\right)\right]
\end{aligned}
$$

where in writing the second expression above we have defined a new parameter $\hat{a}\left(\hat{\lambda}_{\text {eff }}\right) \equiv$ $b_{p} \hat{\lambda}_{\text {eff }}^{(p-3) /(5-p)}$. The quantity $\hat{a}$, as has been mentioned for the ordinary Yang-Mills theory, characterizes the number of degrees of freedom at temperature $T$ since both the energy density and the entropy density can be seen to be proportional to it [29]. Thus by comparing the second expression of (38) with (25), we find that the dependence of the jet quenching parameter on the coupling constant, the temperature and the noncommutativity parameter is indeed universal. This has been noted for $(p+1)$-dimensional ordinary Yang-Mills theory in [29] and we see here that it holds for $(p+1)$-dimensional NCYM theory as well. From (38), we notice that when $\theta=0$, we recover precisely the commutative result obtained in [29, 34]. Non-commutativity reduces the value of the jet quenching parameter from its commutative value. On the other hand for large non-commutativity we get,

$$
\begin{aligned}
\hat{q}_{\mathrm{NCYM}}= & \frac{(9-p) \Gamma\left(\frac{6-p}{7-p}\right)}{\pi^{\frac{3}{2}} \Gamma\left(\frac{5-p}{14-2 p}\right)} \frac{1}{b_{p}^{\frac{1}{2}} \hat{\lambda}_{\mathrm{eff}}^{\frac{p-3}{2(5-p)}}(T) \sqrt{\hat{\lambda}_{\mathrm{eff}}(T)} T \theta^{2}} \\
& \times\left[1-\frac{9-p}{8 \pi^{2}} \frac{1}{b_{p} \hat{\lambda}_{\mathrm{eff}}^{\frac{p-3}{5-p}}(T) \hat{\lambda}_{\mathrm{eff}}(T) T^{4} \theta^{2}}+O\left(\frac{1}{\theta^{4}}\right)\right] \\
= & \frac{(9-p) \Gamma\left(\frac{6-p}{7-p}\right)}{\pi^{\frac{3}{2}} \Gamma\left(\frac{5-p}{14-2 p}\right)} \frac{1}{\sqrt{\hat{a}\left(\hat{\lambda}_{\mathrm{eff}}\right)} \sqrt{\hat{\lambda}_{\mathrm{eff}}(T)} T \theta^{2}} \\
& \times\left[1-\frac{9-p}{8 \pi^{2}} \frac{1}{\hat{a}\left(\hat{\lambda}_{\mathrm{eff}}\right) \hat{\lambda}_{\mathrm{eff}}(T) T^{4} \theta^{2}}+O\left(\frac{1}{\theta^{4}}\right)\right]
\end{aligned}
$$

Again comparing the second expression of (39) with (26), we find that the jet quenching parameter has a universal structure for all $p$. 


\section{Conclusion}

To conclude, in this paper we have calculated the jet quenching parameter of the plasma of $(3+1)$-dimensional NCYM theory using gauge/gravity duality. We used the fundamental string as a probe in the background of (D1, D3) bound state system in the NCYM decoupling limit and extremized the Nambu-Goto action in a particular light-cone gauge. This by gauge/gravity duality is related to a light-like Wilson loop of the NCYM theory where the boundary of the string world-sheet is the loop in question. From the Wilson loop we extracted the jet quenching parameter. However, to obtain its value we had to regularize an integral. This is on top of another regularization that is usually performed in the commutative case corresponding to the self-energy of the quark-antiquark pair. In the non-commutative case another regularization is necessary because here the NCYM theory does not live at the usual boundary $r=\infty$, but at a finite distance before $r=\infty$ [20]. Once we remove the divergent term, NCYM theory can be considered to be living on $r=\infty$ boundary. We gave its expression for both small and large non-commutativity. For small non-commutativity the jet quenching parameter gets reduced in value from its commutative counterpart as non-commutativity introduces a non-locality in space and smoothes out the interaction. The correction term is proportional to $T^{7}$ and so the reduction gets enhanced with increasing temperature as $T^{7}$. We have also estimated the correction in the jet quenching parameter due to non-commutativity by taking into account the experimental bound on the non-commutativity scale $\theta$. We found that it would be difficult to find any significant contribution due to non-commutativity at the present collider energy both at RHIC or at LHC. So, the contribution due to non-commutativity will be significant only at much higher energies. We have generalized our results for plasma of NCYM theories in diverse dimensions. We found that the jet quenching parameter typically depends on $p$, however, it has a universal structure if we rewrite it in terms of a quantity $\hat{a}\left(\hat{\lambda}_{\text {eff }}\right)$, which characterizes the number of degrees of freedom at temperature $T$.

\section{Acknowledgements}

One of us (SR) would like to thank Kamal L Panigrahi for discussion and collaboration at an early stage of this work. We would also like to thank Munshi G Mustafa for useful discussions. We wish to thank the anonymous referee for comments which helped us, we hope, to improve the manuscript. 


\section{References}

[1] M. Gyulassy, X. -n. Wang, Nucl. Phys. B420, 583-614 (1994). nucl-th/9306003; R. Baier, Y. L. Dokshitzer, A. H. Mueller, S. Peigne, D. Schiff, in nuclei," Nucl. Phys. B484, 265-282 (1997). arXiv:hep-ph/9608322 [hep-ph]]; B. G. Zakharov, JETP Lett. 65, 615-620 (1997). arXiv:hep-ph/9704255 [hep-ph]].

[2] R. Baier, D. Schiff, B. G. Zakharov, Ann. Rev. Nucl. Part. Sci. 50, 37-69 (2000). hep-ph/0002198; ; A. Kovner, U. A. Wiedemann, hep-ph/0304151; M. Gyulassy, I. Vitev, X. -N. Wang, B. -W. Zhang, nucl-th/0302077. P. Jacobs, X. -N. Wang, Prog. Part. Nucl. Phys. 54, 443-534 (2005). hep-ph/0405125]; J. Casalderrey-Solana, C. A. Salgado, Acta Phys. Polon. B38, 3731-3794 (2007). arXiv:0712.3443 [hep-ph]]; A. Accardi, F. Arleo, W. K. Brooks, D. D'Enterria, V. Muccifora, Riv. Nuovo Cim. 32, 439-553 (2010). arXiv:0907.3534 [nucl-th]]; U. A. Wiedemann, arXiv:0908.2306 [hep-ph]]; A. Majumder, M. Van Leeuwen, arXiv:1002.2206 [hep-ph]]; J. CasalderreySolana, H. Liu, D. Mateos, K. Rajagopal, U. A. Wiedemann, arXiv:1101.0618 [hepth]].

[3] K. J. Eskola, H. Honkanen, C. A. Salgado, U. A. Wiedemann, Nucl. Phys. A747, 511-529 (2005). [hep-ph/0406319]; A. Dainese, C. Loizides, G. Paic, Eur. Phys. J. C38, 461-474 (2005). hep-ph/0406201; P. F. Kolb, U. W. Heinz, nucl-th/0305084.

[4] E. V. Shuryak, Nucl. Phys. A750, $64-83$ (2005). hep-ph/0405066].

[5] M. Asakawa, T. Hatsuda, Phys. Rev. Lett. 92, 012001 (2004). hep-lat/0308034; S. Datta, F. Karsch, P. Petreczky, I. Wetzorke, Phys. Rev. D69, 094507 (2004). hep-lat/0312037.

[6] H. Liu, K. Rajagopal, U. A. Wiedemann, Phys. Rev. Lett. 97, 182301 (2006). hep-ph/0605178.

[7] J. M. Maldacena, Adv. Theor. Math. Phys. 2, 231-252 (1998). hep-th/9711200; E. Witten, Adv. Theor. Math. Phys. 2, 253-291 (1998). [hep-th/9802150]; S. S. Gubser, I. R. Klebanov, A. M. Polyakov, Phys. Lett. B428, 105-114 (1998). hep-th/9802109; O. Aharony, S. S. Gubser, J. M. Maldacena, H. Ooguri, Y. Oz, Phys. Rept. 323, 183-386 (2000). hep-th/9905111); E. Witten, Adv. Theor. Math. Phys. 2, 505-532 (1998). [hep-th/9803131]. 
[8] J. M. Maldacena, Phys. Rev. Lett. 80, 4859-4862 (1998). hep-th/9803002; S. J. Rey, J. -T. Yee, Eur. Phys. J. C22, 379-394 (2001). hep-th/9803001); S. J. Rey, S. Theisen, J. -T. Yee, Nucl. Phys. B527, 171-186 (1998). [hep-th/9803135]; A. Brandhuber, N. Itzhaki, J. Sonnenschein, S. Yankielowicz, Phys. Lett. B434, 36-40 (1998). [hep-th/9803137].

[9] C. P. Herzog, A. Karch, P. Kovtun, C. Kozcaz, L. G. Yaffe, JHEP 0607, 013 (2006). hep-th/0605158; A. Buchel, Phys. Rev. D74, 046006 (2006). hep-th/0605178); E. Caceres, A. Guijosa, JHEP 0612, 068 (2006). hep-th/0606134); F. -L. Lin, T. Matsuo, Phys. Lett. B641, 45-49 (2006). hep-th/0606136]; S. D. Avramis, K. Sfetsos, JHEP 0701, 065 (2007). hep-th/0606190]; N. Armesto, J. D. Edelstein, J. Mas, JHEP 0609, 039 (2006). [hep-ph/0606245]; E. Nakano, S. Teraguchi, W. Y. Wen, Phys. Rev. D75, 085016 (2007). hep-ph/0608274]; G. Bertoldi, F. Bigazzi, A. L. Cotrone, J. D. Edelstein, Phys. Rev. D76, 065007 (2007). [hep-th/0702225]; F. Bigazzi, A. L. Cotrone, J. Mas, A. Paredes, A. V. Ramallo, J. Tarrio, JHEP 0911, 117 (2009). arXiv:0909.2865 [hep-th]].

[10] J. Gomis, T. Mehen, Nucl. Phys. B591, 265-276 (2000). hep-th/0005129.

[11] W. Heisenberg, W. Pauli, Z. Phys. 56, 1-61 (1929); Z. Phys. 59, 168-190 (1930).

[12] H. S. Snyder, Phys. Rev. 71, 38-41 (1947); Phys. Rev. 72, 68-71 (1947).

[13] A. Connes, in * Cargese 1987, Proceedings, Nonperturbative Quantum Field Theory* 33-69; "Noncommutative Geometry", Academic Press, San Diego, CA (1994).

[14] A. H. Chamseddine, A. Connes, Phys. Rev. Lett. 77, 4868-4871 (1996).

[15] S. M. Carroll, J. A. Harvey, V. A. Kostelecky, C. D. Lane, T. Okamoto, Phys. Rev. Lett. 87, 141601 (2001). hep-th/0105082.

[16] A. Anisimov, T. Banks, M. Dine, M. Graesser, Phys. Rev. D65, 085032 (2002). hep-ph/0106356.

[17] I. Mocioiu, M. Pospelov, R. Roiban, Phys. Lett. B489, 390-396 (2000). hep-ph/0005191.

[18] S. Doplicher, K. Fredenhagen, J. E. Roberts, Commun. Math. Phys. 172, 187-220 (1995). hep-th/0303037.

[19] N. Seiberg, E. Witten, JHEP 9909, 032 (1999). hep-th/9908142. 
[20] J. M. Maldacena, J. G. Russo, JHEP 9909, 025 (1999). [hep-th/9908134].

[21] A. Hashimoto, N. Itzhaki, Phys. Lett. B465, 142-147 (1999). hep-th/9907166.

[22] A. Connes, M. R. Douglas, A. S. Schwarz, JHEP 9802, 003 (1998). hep-th/9711162.

[23] J. C. Breckenridge, G. Michaud, R. C. Myers, Phys. Rev. D55, 6438-6446 (1997). hep-th/9611174]; M. S. Costa, G. Papadopoulos, Nucl. Phys. B510, 217-231 (1998). hep-th/9612204]; J. G. Russo, A. A. Tseytlin, Nucl. Phys. B490, 121-144 (1997). hep-th/9611047.

[24] C. -S. Chu, P. -M. Ho, Nucl. Phys. B550, 151-168 (1999). hep-th/9812219; F. Ardalan, H. Arfaei, M. M. Sheikh-Jabbari, Nucl. Phys. B576, 578-596 (2000). hep-th/9906161.

[25] J. Sadeghi, B. Pourhassan, arXiv:1002.1596 [hep-th]].

[26] M. Ali-Akbari, [arXiv:1104.4924 [hep-th]].

[27] K. L. Panigrahi, S. Roy, JHEP 1004, 003 (2010). arXiv:1001.2904 [hep-th]].

[28] R. -G. Cai, N. Ohta, JHEP 0003, 009 (2000). hep-th/0001213.

[29] H. Liu, K. Rajagopal, U. A. Wiedemann, JHEP 0703, 066 (2007). hep-ph/0612168.

[30] A. Dhar and Y. Kitazawa, Phys. Rev. D 63, 125005 (2001) hep-th/0010256].

[31] M. Alishahiha, Y. Oz, M. M. Sheikh-Jabbari, JHEP 9911, 007 (1999). hep-th/9909215.

[32] S. Roy, Phys. Lett. B682, 93-97 (2009). arXiv:0907.0333 [hep-th]].

[33] N. Itzhaki, J. M. Maldacena, J. Sonnenschein, S. Yankielowicz, Phys. Rev. D58, 046004 (1998). hep-th/9802042].

[34] S. Chakraborty, S. Roy, Nucl. Phys. B850, 463-476 (2011). arXiv:1103.1248 [hepth]]. 